\title{
Null and Noteworthy: Quality of life, IACC discussion, mixed results on interventions
}

\author{
BY LAURA DATTARO
}

2 DECEMBER 2021

Last month, the Interagency Autism Coordinating Committee (IACC) met for the second time this year. Part of their discussion touched on how to make selections for their Summary of Advances, a list of studies given to policymakers to represent "groundbreaking and significant progress" in the field.

This led to an important question: What counts as groundbreaking and significant? One committee member, Paul Wang of the Simons Foundation, wondered if it might be valuable to include not only significant positive findings, but also those that found null results, or, as he called them, "papers that perhaps show we do not have an answer." (The Simons Foundation is Spectrum's parent organization.) "Can we recognize these, you might call them negative results, as important publications?" Wang asked.

IACC is still putting together its list, but here at Null and Noteworthy we routinely recognize those results. Spectrum has reported on several recently, including an intranasal oxytocin trial that found no benefit for autistic children and two studies rebutting a proposed association between labor epidurals and autism; a third is discussed below, along with other results.

Thanks, as always, for your feedback, and please continue to send your thoughts, ideas, interesting studies and cat photos to laura@spectrumnews.org.

\section{Epidurals, one more time:}

A previous edition of this newsletter highlighted a rebuttal to a study that found increased odds of autism in children born to women who had received an epidural during labor. Two more studies, published in September, echoed the null results. Now, yet another study brings the null total to four. This one used data from the Scottish National Health service to examine, among other things, neurodevelopmental outcomes of 435,281 children born in Scotland between 2007 and 2016. 
After adjusting for confounding factors, the researchers found that children born to women who had received an epidural were less likely to have problems with communication or fine motor skills at age 2 than children whose mothers had not received an epidural. An editorial accompanying the study noted that the findings provide further evidence that there is "no meaningful association" between epidurals and neurodevelopmental conditions in children.

The findings and editorial were published in JAMA Network Open in October.

\section{Which intervention for whom?:}

Much autism research is predicated on the idea that providing interventions early in life - possibly before autism is diagnosed - results in better outcomes for the child. But a recent review demonstrates that the reality is not so straightforward. The researchers analyzed results from 13 studies of parent-provided interventions for 715 infants and toddlers with high odds of being autistic. Children who received interventions did not show any significant improvements in their language, social behavior, motor skills or other developmental outcomes, though some did show small changes in their autism traits. The findings demonstrate that researchers still don't know which interventions work best for which children, the authors write, identifying an "urgent need" for a more nuanced approach to studying and implementing early interventions.

The findings were published in Autism in October.

\section{Confirmation collection:}

Because science is never straightforward, another review on interventions came to the opposite conclusion. This one analyzed studies attempting to replicate a 1987 study showing the effectiveness of an applied behavior analysis (ABA) therapy designed by the University of California, Los Angeles (UCLA) Young Autism Project and first introduced in 1980; the study has been cited nearly 70,000 times. The new work identified 17 studies involving 595 autistic children, 401 of whom participated in UCLA intervention groups (the remaining children did not receive interventions). On average, children in the UCLA groups showed fewer autism traits over time, a change not seen in controls, replicating the initial findings. The intervention also appeared to help with cognitive function and communication skills.

The findings were published in Behavioral Interventions on 2 November.

\section{Quality of life:}

If autism research aims to improve autistic people's quality of life, researchers must first understand how quality of life varies among autistic people. Several studies have shown that autistic women tend to report worse quality of life than autistic men, particularly when it comes to 


\section{Spectrum | Autism Research News}

https://www.spectrumnews.org

their physical health. A new analysis of 67 autistic and 66 non-autistic adults replicated that finding: Whereas both autistic men and women had reduced mental health quality of life compared with non-autistic people, only autistic women reported more physical problems than their nonautistic peers. The researchers also found, however, that autistic women's mental health appeared to improve with age.

The findings were published in Quality of Life Research in October.

\section{Brain folds, revisited:}

The first edition of this newsletter highlighted a review of studies examining cortical gyrification the pattern of folds in the tissue of the cortex - in people with autism or attention deficit hyperactivity disorder (ADHD). Although the review found no differences between the groups, the investigators pointed out that a conclusion could not be reached without using the same methodology to compare people with autism versus ADHD. Now the researchers have done just that. After imaging the brains of 197 autistic children, 96 children with ADHD and 246 neurotypical children, the researchers still found no differences in brain folds among the groups. What's more, the patterns were not associated with any ADHD or autism traits, suggesting that atypical gyrification is not a marker of either condition.

The findings were published in Cerebral Cortex in September.

\section{Et al.:}

- A review of 52 papers found that children born before 37 weeks of gestation are 3.3 times as likely to have autism as children born full-term are, confirming an association between autism and premature birth.

- People with an autism-linked variant in chromosome 16 sleep for just as long as those without the variant, though they do have more disturbed sleep.

- Neither autistic people nor those with elevated autism traits have distorted feedback loops involving sensory input and expectations, a problem seen in people with schizophrenia, according to a small study on such 'circular inference.'

- Sequencing newborns' genomes does not cause additional stress for their parents, even if the sequencing turns up a genetic risk, according to a survey of the extended-screening program BabySeq; Spectrum wrote about the push for such screens for autism-related conditions in July.

- Autistic and non-autistic children show no differences in the motor functions that control eye movements, suggesting those functions can't account for observed eye-gaze differences in people with autism.

- How children born preterm play does not correlate with those children's autism traits, in contrast with the play of siblings of autistic children, even though both groups have an elevated likelihood of having autism. 


\section{Spectrum | Autism Research News}

https://www.spectrumnews.org

- Antipsychotics don't increase the risk of seizures in people with autism, who are frequently prescribed the drugs.

- In both autistic and non-autistic children, anxiety symptoms are not linked to amygdala volume, despite other research suggesting links between the amygdala and anxiety.

- A review of interventions designed to improve executive function in autistic children and young adults noted that a lack of replication studies and under-publishing of null results clouded the conclusions.

- A small study of motor learning found that people with and without autism are equally adept at adjusting their keyboard taps in response to unpredictable stimuli.

- And finally, the Ritvo Autism Asperger's Diagnostic Scale does not appear to be a useful screener for autism in adults, at least according to one small study.

Cite this article: https://doi.org/10.53053/QRJQ1978 